\title{
Compression of visual space in natural scenes and in their photographic counterparts
}

\author{
HEIKO HECHT \\ Universität Bielefeld, Bielefeld, Germany \\ and \\ ANDREA VAN DOORN and JAN J. KOENDERINK \\ Universiteit Utrecht, Utrecht, The Netherlands
}

\begin{abstract}
Classical theories of space perception posit continuous distortions of subjective space. These stand in contrast to the quantitatively and qualitatively different distortions experienced in space that is represented pictorially. We challenge several aspects of these theories. Comparing real-world objects with depictions of the same objects, we investigated to what extent distortions are introduced by the photographic medium. Corners of irregularly shaped buildings had to be judged in terms of the vertical dihedral angles subtended by two adjacent walls. Across all conditions, a robust effect of viewing distance was found: Building corners appear to flatten out with distance. Moreover, depictions of corners produce remarkably similar results and should not receive a different theoretical treatment than do real-world scenes. The flattening of vertical angles cannot be explained by a linear distortion of the entire visual space. We suggest that, for natural scenes, compression of space is local and dependent on contextual information.
\end{abstract}

They [space relations] are nothing but sensations of particular lines, particular angles, particular forms of transitions. (James, 1890/1950, p. 152)

Visually perceived depth in spatial arrangements has been studied within the context of two rather distinct frameworks. First, many theories of subjective space are based on the notion that perceived space is distorted in a uniform fashion and that a specific transformation can be found that describes the mapping relations between the Euclidean space of the world and our subjective space. Second, theories of spatial representation are concerned with distortions that arise in the process of representing space-mainly, the effects caused by pictorial rendition, such as projective distortions or the apparent flattening of the scene. In this study, we attempt to establish a relationship between the two frameworks by comparing judgments made in real scenes with judgments based on photographic renditions of the same scenes. Surprisingly, we found distortions to be very similar. We had observers judge the dihedral angles of building corners from a va-

H.H.'s work was supported by the Deutsche Forschungsgemeinschaft (He 2122/4, He 2122/5). The authors thank the members of the staff and the Forschungsgruppe "Perception and Evolution" at the Zentrum für interdisziplinäre Forschung in Bielefeld, as well as the members of the Utrecht physics department who volunteered their time. We also thank Stephen Ellis, Zygmunt Pizlo, and two anonymous reviewers for their insightful comments. Correspondence concerning this article should be addressed to $\mathrm{H}$. Hecht, $\mathrm{ZiF} /$ Universität Bielefeld, Wellenberg 1 , 33615 Bielefeld, Germany (e-mail; hecht@hrz.uni-bielefeld.de).

-Accepted by previous editor, Myron L. Braunstein riety of distances and viewpoints. In both real-world and photographic viewing from the appropriate eye point, the angles appeared flatter at larger distances. If we had looked at pictures alone, these findings could be interpreted as flattening effects that are due to the nature of photographs. However, we will show that rendition is secondary and that a compression of subjective space along the sagittal plane exists regardless of viewing mode.

For centuries, the camera metaphor of perception has prompted philosophers to believe that an explanatory gap exists between the more or less two-dimensional (2-D) pictures on our retinas and our ability to perceive distances and depth relations in the three-dimensional (3-D) world. In Descartes' time, an extraretinal vergence signal was thought to fill the gap and to provide the necessary and sufficient information for accurate depth judgments (see Foley, 1980). Later, disparity and parallax (Helmholtz, 1867) were deemed necessary to explain our abilities. This was the beginning of the development of a very productive field of research that mainly collected cues that affect apparent depth (for a recent summary, see Cutting \& Vishton, 1995). Attempts to integrate the effects of these cues have not (yet) been pieced together into a general theory of perceived depth. And despite elaborate weighting models of cue integration (e.g., Young, Landy, \& Maloney, 1993), it may not even be possible at all. Astonishingly, rather independently of this development, many researchers believe in a lawful distortion of visual space and found their beliefs on or in opposition to Luneburg's (1947) theory of visual space. The following paragraphs give a paradigmatic overview of these ideas and try to show that the narrowly defined method and stimuli 
that are used in this context may explain the consistent findings. We deviated from this tradition and used large objects as stimuli and direct angle judgments as method. Then, a seemingly unrelated group of effects will be mentioned that have only been related to picture perception. Finally, some hypotheses will be derived and tested.

\section{DISTORTIONS OF SUBJECTIVE VISUAL SPACE}

Theories of perceptual visual space have taken a peculiar development that could be attributed to a traditional emphasis on vergence and stereo information and to the exclusive use of rather reduced stimuli to test these theories. Before Helmholtz (1867), the extraretinal information provided by vergence alone was believed to allow us to judge distance accurately, with some imprecision entering at large distances (Foley, 1980). In contrast, Helmholtz reported that observers suffer systematic distortions even when objects are presented binocularly at close range. He proposed that sensations of distance may not be veridical because the extraretinal vergence signal is not always processed reliably. For instance, the system might underestimate the convergence of the visual axes because observers overestimate the distance of near targets for other reasons.

Helmholtz (1867) proposed parallax and effective disparity between the retinal images as possible reasons for misjudgments of egocentric distance. In his classic experiment, he used three threads, two in equidistant fixed positions and a center one that could be adjusted in depth. Adjustments were made so that all the threads appeared to lie in one frontoparallel plane. Observers produced apparent coplanar arrangements that deviated systematically from true coplanarity; the center thread was adjusted too close for large viewing distances and too far for short viewing distances. Put differently, for short viewing distances, vertical thin objects appeared to be on a frontoparallel plane, when they were, in fact, on a concavely curved surface. This result cannot be explained by vergence, but disparity might be responsible. And in fact, subjective curvature of straight objects had been known long before. For instance, R. Smith (1738) and Hauck (1875, both cited in Pirenne, 1970) reported that a view down a long boulevard gave the impression that its sides did not recede in parallel straight lines but that it was overly narrow close to the observer and overly wide farther away.

\section{Horopter Theory and the Apparent Frontoparallel Plane}

If objects that fall on corresponding positions of the two retinas are perceived to be coplanar, then depending on the point of fixation, a straight frontoparallel arrangement of objects should look curved. This we will call the horopter theory of subjective space curvature. Thus, objects are thought to appear to be coplanar and, possibly, at the same egocentric distance from the observer (Fry, 1950; Ogle, 1950), if they fall on corresponding retinal locations. To our knowledge, the theoretical gap between retinal correspondence and phenomenal curvature has never been bridged satisfactorily, and most researchers fail to hypothesize why the horopter should influence the perceived frontoparallel arrangement. Prima facie, such an influence is not very plausible, and after all, when looking at a wall in front of us, we are aware that the far corners are radially farther away from us than the center that we fixate. Moreover, the results only hold for viewing distances of less than $100 \mathrm{~cm}$.

The theoretical situation becomes even less satisfactory because the perceived frontoparallel arrangement is never on the Vieth-Müller circle, but somewhere between it and the true frontoparallel. The concept of empirical horopter (Howard \& Rogers, 1995) was introduced to quantify deviations between the horopter and the perceived frontoparallel arrangement, which is also called the Hering-Hillebrand deviation. A look at this deviation is justified for close viewing distances, because the empirical horopter differs from the true frontoparallel toward the theoretical horopter (Vieth-Müller circle). ${ }^{1}$ However, for viewing distances of more than 2 or $3 \mathrm{~m}$, the situation is the reverse: The subjective frontoparallel becomes convex - that is, it curves away from the horopter. It is incomprehensible how horopter theory could have been maintained despite these data, which were obtained consistently (e.g., Ames, Ogle, \& Glidden, 1932; Blumenfeld, 1913). Also, some doubts about the universality of the curved subjective frontoparallel were voiced very early on. Helmholtz (1867; Lukas, 1996) noticed that the effect disappeared as soon as the threads that he used were modified - for example, by adding little beads.

\section{Alley Experiments: \\ Subjective Curvature Is Task Dependent}

Following Helmholtz (1867), the basic results have been replicated innumerable times, with variations that resulted in three different theories describing how the geometry of visual space deviates from Euclidean space. Besides Helmholtz, the first to suggest that visual space is non-Euclidean was Blumenfeld (1913). When experimenting on size-distance invariance, he discovered that apparently straight arrangements of lights not only curved, they did so differently, depending on how they were constructed by the observer. In the parallel alley task, two rows of lights (e.g., symbolizing street lights as seen when looking straight down a street) have to be adjusted so that the lights appear to be evenly spaced from one to the next on either side of the street. With this method, the produced distances between the closest lights on opposite sides of the street (left to right) were smaller than those between two corresponding lights farther away. In the distance alley task, on the other hand, observers have to adjust corresponding lights (on the left and the right side of the street) so that they appear to be separated by equal distances from each other. In this case, when looking at the street as a whole, it appears to diverge with distance. These results already suggest that visual space 
cannot be Euclidean (for summaries, see Lukas, 1996; Suppes, 1977).

Parallel and distance alleys, usually produced by rows of adjustable point lights, became the method of choice for a large number of experiments on subjective space, only a few of which are mentioned here. Extending Blumenfeld's (1913) results, Luneburg (1947) formulated a metric for visual space, suggesting that it is best described by a Riemannian geometry of constant Gaussian curvature. To this date, this geometry is the subject of many research projects and elaborations (e.g., Indow, 1991; Indow, Inoue, \& Matsushima, 1962a, 1962b; Indow \& Watanabe, 1988). In parallel, Luneburg's metric was refined by Blank (1961), whose motivation was to discard parameters that might reflect extraretinal information, such as vergence. He tested whether subjective space is curved hyperbolically or elliptically. Observers saw three starlike lights forming an isosceles triangle, with two points located $71 \mathrm{~cm}$ and the third $274 \mathrm{~cm}$ in front of the observer. He or she had to place a fourth light where it appeared to bisect the left (or right) side of the triangle. This technique is able to rule out effects caused by sagittal compression, which does not affect the projected lateral bisection points observers had to judge. The points were, on average, moved inside the triangle, which indicates hyperbolic curvature of visual space.

Foley $(1972,1977,1980)$ put forth a third geometry of visual space, explicitly incorporating parameters that could reflect vergence information and explain absolute and relative misperception of distances. His results led him to conclude that visual space is not only non-Euclidean, but also nonhomogeneous. In a decisive experiment, Lukas (1983) suggested that, among the three geometries, the original one proposed by Luneburg (1947) provided the best fit for laboratory data. He produced point lights with an oscilloscope and presented them in a mirror stereoscope at simulated distances of between 25 and $1,000 \mathrm{~cm}$.

\section{Introducing Natural Viewing Conditions and the Problem of Context Effects}

Point lights on uniform backgrounds dominated the research on subjective space; however, there are some studies performed in the outdoors with partially diverging results. Alley and horopter experiments, as well as experiments with three point lights, have been replicated under natural viewing conditions. Zajaczkowska (1956) was the first to allow her observers free eye movements and found subjective flattening at larger distances. However, the effect cannot be attributed to eye movements, since they are practically ineffective at large distances (Foley, 1980).

On the basis of the results of an outdoor experiment, Battro, Di Pierro Netto, and Rozestraten (1976) suggested that the curvature of the apparent frontoparallel plane found in horopter experiments (convex curvature at large viewing distances) was an artifact of the method used. Their observers had to tell the experimenter how to arrange thin stakes on the grassy terrains of polo fields so that they would lie on a frontoparallel plane. When they used the Helmholtz method (adjust the center stake), convex curvature was found for large distances (up to $70 \mathrm{~m}$ ) - that is, the laboratory data were replicated. Then Battro et al. had some other observers direct the arrangement of stakes to the left and right of their line of sight while the center stakes remained fixed (Ogle's [1950] method). Here, concave curvature was found at large distances. For both methods, the respective curvature reversed at close distances. It is likely, however, that the differences ascribed to the Ogle method are due to context effects created by uncontrolled features of the terrain, since the classical results have been replicated numerous times (Indow, 1991, 1997). Another finding remains uncontested. Battro et al. showed visual space to be scale dependent. Distortions increase with the distance of the coplanar stimuli from the observer.

Helmholtz (1867), as well as a vast number of experimenters to follow, used mostly point lights or thin threads as stimuli, which were presented in the dark or in front of a uniform background. All the theories of subjective space (with the possible exception of that of Gibson, who believed that we have direct access to the Euclidean space of the world) suggested lawful distortions of subjective space on the basis of findings with these rather diminished stimuli. Richer variables, such as texture gradients and texture regularity (Gibson, 1950), projected size (Gilinsky, 1951; Gogel, 1964; W. M. Smith, 1952), and motion parallax (Braunstein, 1968), were only studied much later, and usually not with the goal of testing theories of visual space. Thus, to this date, subjective space and its alleged distortions are based on a small set of point stimuli or of narrow vertical lines on a uniform terrain. This prompted us to use more richly textured real-world objects as stimuli.

Context effects have, nonetheless, been subject to investigation and, in one case, even within a framework of visual space. Gogel (1993), who attempted to study perceived space in purely phenomenal terms, perfected a method that involved observers fixating a target. A laterally movable rod close to the observer had to be aligned with a stationary rod in front of the target. Depending on the alignment, the perceived distance of the target could be measured. Filling a scene with different targets and measuring their respective perceived distances allowed a reconstruction of phenomenal space. Two main findings were obtained. First, all the objects seemed to be displaced by a distance of $2-3 \mathrm{~m}$ from the observer (specific distance tendency). Second, there was a tendency to perceive objects as being evenly spaced (equidistance tendency) and the distances between them as being compressed (Gogel, 1965). Gogel believed that phenomenal geometry is usually internally consistent, and that when it is not, this is most likely because of cognitive influences.

\section{Conclusion for Visual Space}

The research of the last 100 years on binocular subjective space, as summarized above, differs vastly as a func- 
tion of the methods that were used. Roughly, experiments involving the production of frontoparallel planes found curvatures, corresponding to a compression of space along the line of sight, as compared with lateral locations. Alley experiments involving multiple adjustments across sides, rather than along the length of the road, yielded different subjective curvatures, thus suggesting that visual space cannot be Euclidean. And recently, many researchers seemingly have abandoned the notion of a uniformly distorted subjective space. Foley (1980) has advocated nonhomogeneous space for a long time, and Indow $(1991,1997)$ has focused on the contextual effects of perceived space. Also, Lukas (1996), who collected new data on three- and four-point light arrangements, revised his old (Lukas, 1983) conclusion, which agreed with Luneburg $(1947,1950)$, that visual space is uniformly curved, in favor of a nonhomogeneously distorted space, in the sense of Foley (1972).

\section{DEPTH COMPRESSION}

The existing theories of 3-D space perception that attempt to explain subjective visual distortions in the layout of our action space (e.g., Gogel, 1964; Luneburg, 1950) are based on laboratory effects. ${ }^{2}$ And there our visual grasp of spatial relationships is remarkably good, notwithstanding some systematic distortions. However, we will argue that the exceptions mentioned so far (e.g., Battro et al., 1976) should be taken seriously. For example, very large objects, such as roads or hill sites, are able to fool our senses quite dramatically and appear to belong to a different class of phenomena, as compared with the relatively small distortions found in laboratory experiments. Objects that are too large to be manipulated directly or with classical personal tools appear to be treated differently by our visual system. Likewise, objects that are far away from us appear to be subject to rather strong visual distortions (Ross, 1974).

In an outdoors experiment, again using thin stakes positioned in large grassy fields, Wagner (1985) found that observers make dramatic judgment errors. Among other things, they had to judge the angles subtended between the corners of triangles, which were indicated by three randomly placed stakes. Sagittal depth was found to be substantially compressed by more than $50 \%$. Likewise, Loomis, Da Silva, Philbeck, and Fukusima (1996) found depth compression of up to $90 \%$ in a task in which observers had to match the sagittal distance between two objects to a given frontoparallel distance. The effect increased with distance from the observer. Interestingly, blindfolded walking based on prior visual information was not subject to the same compression. Watanabe (1996), however, found some distortion of walking space and argued that our visual space is hyperbolic, whereas our walking space is elliptic. He asked blindfolded subjects to turn right by $45^{\circ}$ and then walk straight for $4 \mathrm{~m}$. They did not stay on a straight path, as would be predicted by a Euclidean representation of subjective space, but deviated and ended up on the left side of the median line, which is consistent with an elliptic space. When observers adjusted visual pointers, on the other hand, evidence for hyperbolic visual space was found. It is not clear, however, to what extent these results could be a function of slight asymmetries of the motor system, in the case of blindfolded walking. As soon as one limb is stronger than the other, one would expect curved paths as an outcome of the intention to walk straight.

A compression of visual space, be it hyperbolic or less regularly structured, also seems to be one of the few commonalties of the numerous studies performed on parallel and distance alleys. Unfortunately, compression and perceived absolute distances often were not measured. For example, in Blank's (1961) experiment, it was never assessed whether the farther of the three triangularly arranged point lights appeared closer than it actually was. This alone would explain his results' conforming with a hyperbolic distortion. A notable exception is the outdoors study by Wagner (1985), described earlier. Compression or foreshortening has also been reported as a result of reduced and monocular cues. Foley (1977) found perfect distance estimates for the close range of up to $40 \mathrm{~cm}$ in multicue conditions, but linear distortion (foreshortening) in monocular viewing.

Horopter studies suggest a curvature of space that is not always compatible with the notion of spatial compression. Sagittal compression would predict that corners or angles would flatten out regardless of whether they are convex or concave. Horopter theory, on the other hand, can be taken to predict different effects for convex and for concave corners. For instance, at a close viewing distance, concave angles should be perceived as less acute than they are, and convex corners as more acute - that is, only concave corners should flatten.

\section{SURFACE SLANT PERCEPTION}

A rather large body of research that implicitly relates to perceived subjective space can be found in the literature on surface slant perception. When observers are asked to determine the orientation of a displayed object with respect to their line of sight, they typically perceive it too close to the frontoparallel plane (see, e.g., Flock, 1964; Gibson \& Cornsweet, 1952; Gillam \& Ryan, 1992). That is, despite informative cues of texture, perspective, and disparity, optical slant perception is biased toward the frontoparallel. These results are certainly in tune with the notion of general depth compression. Similarly, geographical slant is often misjudged toward the environmental vertical. Geographical slant is defined as the inclination of a surface-say, a hill site-with respect to the environmental horizontal, which is typically the ground plane. Thus, a geographical slant of $0^{\circ}$ would be horizontal. It is rather surprising that the literature on surface slant has never been explicitly related to theories of subjective space. This could be easily done via the ubiquitous finding of depth compression. 
Although geographical slant estimation does not involve a direct comparison of depth relations, many findings could be rephrased in terms of depth compression. For example, Proffitt, Bhalla, Gossweiler, and Midgett (1995) found that geographical slant is often overestimated by $100 \%$ and more. An assumed depth compression of $50 \%$ would nicely explain this finding. Take a hill sloped at, say, $30 \%$, which is equal to a slant of $16.7^{\circ}$. Were we to underestimate the depth of the hill's base by $50 \%$, the resulting slope would be $31^{\circ}$, or $60 \%$, which is an overestimation of about $100 \%$. The same calculation done for a concave building corner, whose vertex is at $10 \mathrm{~m}$ from the observer, yields the following result: Assuming only sagittal compression by $50 \%$ and no other distortions, a $90^{\circ}$ angle should look to be $126.8^{\circ}$. We will show that this corresponds roughly to empirical angle overestimations.

\section{PICTORIAL EFFECTS ON PERCEIVED VISUAL SPACE}

There is a striking difference between the tenuous attempts to map perceived space onto physical space in the context of point light worlds and the unwillingness to do anything like it in the context of photographs, which can provide much richer information. A general metric of rendered visual space, to our knowledge, has never been proposed, probably because preliminary attempts to investigate how $2-D$ relations on the projected image influence our 3-D interpretations of it (for an overview, see Hagen, 1980) yielded many contradictory results. To mention a few, there is an amazing robustness of pictorial space, despite sometimes dramatic projective distortion (Pirenne, 1970). It is not settled whether this robustness is best interpreted as an array-specific extraction of depth information (Bengston, Stergios, Ward, \& Jester, 1980; Gibson, 1954), as a lack of discriminability, or as the result of an effective compensation mechanism (Cutting, 1987). Also, the ability to compensate is highly dependent on viewing conditions, such as vantage point. On the other hand, there is evidence for many picture artifacts, such as flattening owing to a truncated visual field (Hagen, Jones, \& Reed, 1978; Lumsden, 1983) or to the frame that is visible around the picture (Eby \& Braunstein, 1995). In other words, the specialty of pictures and a lack of a uniform distortion in pictorial representations is an underlying assumption. Pictures are used successfully to communicate spatial relationships, and it is worthwhile to compare the compression found in real scenes with that found in pictures.

One of the few studies that investigated the geometry of pictorial monocular visual space was carried out by Drösler and Konstanty (1987). They used pictures of natural backgrounds, such as a house, railroad tracks, and so forth, and superimposed point lights and lines on them. Observers had to answer questions about geometric relations between the lights with respect to the frontoparallel and the horizontal planes. The authors concluded that monocular visual space is characterized by nonmetric projections.

O. W. Smith (1958) and Kraft, Patterson, and Mitchell (1986) reported systematic distortions in depth judgments made when viewing photographs of natural scenes. Typically, sagittal depth is foreshortened, whereas lateral width is not. This effect is larger with lenses with large focal lengths and becomes much smaller with wideangle lenses. Much of the effect, however, can be attributed to the truncation of the visual field that correlates with focal length - that is, wide-angle lenses show terrain close to the observer that is invisible in, say, a 50-mm lens (Hagen et al., 1978; Lumsden, 1980).

Ellis, Smith, Grunwald, and McGreevy (1991) have actually compared performance in a direction judgment task in a real-world situation with that in computergenerated displays. Their observers had to judge the azimuth angle between two objects and a reference line that deviated from the line of sight. The objects were positioned below and in front of them. In the computer task, judgment errors were in the direction of projected image directions, indicating a breakdown of position constancy in virtual 3-D space. When the experiment was replicated with observers standing on top of a building and judging angles between objects in the parking lot, the errors were much smaller, indicating that observers were able to make use of the additional information in the natural scene and viewing conditions. The results were also incompatible with the compression of depth found by Wagner (1985). This could be explained by the elevated viewing position that was used by Ellis et al. In a similar exocentric pointing task that placed observers on the ground in an open field, Koenderink, van Doorn, and Lappin (1996) found the classical compression effects. Interestingly, symbolic enhancement of the displays used by Ellis et al. improved performance (Ellis \& Hacisalihzade, 1990), which indicates another layer of complexity when studying renditions of 3-D scenes that use constructed simulated objects, rather than photographed scenes.

What we know about extracting spatial relations from pictures is comparatively little, given how heavily we rely on pictures to represent 3-D spatial layout. Be it reconnaissance, advertising, or virtual reality, we are supposed to extract true depth relations from pictorial renditions. In this study, we take a closer look at the ability to perceive spatial layout from mostly static cues by introducing photographs and, thereby, a pictorial viewing condition that comes as close as possible to the real-world situation.

\section{OVERVIEW OF THE EXPERIMENTS}

It is striking that all the spatial distortions that have been found and used as bases for hypotheses of subjective visual space have employed a very narrow set of stimuli and a narrow set of tasks. With few exceptions, the stimuli were small and isolated. Even in outdoors experiments, small thin objects were presented on large 
uniform terrains. The task of the observer typically involved adjusting stimuli to form apparent planes. Pointing tasks and distance bisection were also used in a few cases. Depending on the task, curvature and/or compression of subjective space are common for larger viewing distances. Laboratory experiments emphasized extra- and interretinal binocular information. Effect sizes of errors of around $10 \%$ are typical for laboratory experiments. Large effects of between $50 \%$ and $100 \%$ were only found outside the laboratory (Wagner, 1985) or in tasks involving reduced cues and memory. For example, Philbeck, Loomis, and Beall (1995) found compression for viewing distances greater than $3 \mathrm{~m}$ (and expansion of visual space for distances less than $3 \mathrm{~m}$ ) when observers had to walk to a target that they could see only before they started walking. The compression was only found for reduced cues. There is also evidence for compression along the sagittal plane of more than $50 \%$ and in full-cue viewing when exocentric judgments have to be made (Loomis et al., 1996).

In our experiments, we attempt to answer a number of questions that can be derived from the current state of research on the topic of viewing conditions and their effect on visual space. First, is compression a function of reduced visual cues? And in particular, is the effect, or some portion of it, produced by the sparse stimuli that are often employed. We hypothesize that stereo and vergence information play no or a very small role in effects of subjective curvature or distance distortion. Second, if there is compression of space, rather than stimulusspecific distortions, the former should also affect objects inside this space and should generalize to stimuli that have not been tested. Thus, large, solid real-world objects (corners of buildings) were chosen as stimuli. Third, if horopter-based curvature generalizes to space, convex and concave corners should be affected differently by space curvature. Fourth, if the classical findings are not artifactual to the methods (Ehrenstein, 1977) used, perceived spatial relations should also be distorted when assessed by a different method. Analogue angle judgments were used in our study. Fifth, many foreshortening effects can be attributed to a very shallow incidence angle between the observer's line of sight and the ground plane (Ellis et al., 1991), which becomes particularly critical for objects presented at large distances on planes with little textural structure. We did not want to study this particular effect and, therefore, chose objects that extended perpendicular to the observer's line of sight-namely, vertical building corners. Finally, are the flattening and compression effects that are found in many pictures caused by the pictorial medium, or can they be fully explained by effects that also hold in natural viewing of the same objects? Consequently, all the stimuli were also presented as photographs.

Our study assessed the ability to make angle judgments of building corners at different viewing distances, ranging from action space at arm's length to distances of about 10 eye heights. The results show that the world appears flatter when we have taken a step back from it. A pilot study established the large size of the effect for a realworld building corner. In Experiment 1, the effect was studied more systematically for convex and concave corners at different viewing angles and distances. Experiment 2 replicated the effect for photographic viewing. To minimize possible influences of conflicting accommodative cues, a large rear projection surface was used.

\section{PILOT STUDY}

Once we direct our attention to a convex corner of a large building in the distance that we know is a right angle, we cannot help but notice that it looks much flatter than $90^{\circ}$. Likewise, concave corners often appear to be more obtuse than they are. We came across a corner that showed this effect rather dramatically, although it was not ideal, since it was somewhat occluded by a tree and some bushes. It is depicted in Figure 1, a photograph taken through a large window from inside a hallway of the Center for Interdisciplinary Research (ZiF) in Bielefeld, Germany. The corner in the center of the photograph, formed by the large concrete wall and the lower windowed wall, looks decisively more obtuse than a right angle. All three authors, as well as some male bystanders, were quite surprised when they went into the courtyard and approached the corner. It turned out that it was, indeed, a right angle. Although right angles are predominant in modern buildings, the $\mathrm{ZiF}$ is highly unusual, insofar as only a small minority of its corners are right angles. Thus, once some time has been spent at the $\mathrm{ZiF}$, right angles will no longer be taken for granted. The pilot experiment was conducted to find out whether observers consistently overestimate the angle subtended by this particular corner.

\section{Method}

Observers. Seven observers ( 1 male, 6 female) participated. They ranged in age from 34 to 58 years. All had normal or correctedto-normal vision and were nave with respect to the purpose of the study. Half of them were paid for their participation.

Stimuli and Design. We chose one vantage point in the courtyard, as close as possible to the window from which the original observation was made. It corresponds to the location from which the photograph of Figure 1 was taken, which was $14.8^{\circ}$ to the right of the bisectrix of the corner. The distance from the vertex was $23.4 \mathrm{~m}$. Then two other vantage points, closer to the corner, were selected. They were on the bisectrix at 15 and $1.5 \mathrm{~m}$, respectively. All three positions can be seen in the schematic drawing shown in Figure 2. Each position was assumed once by all the subjects, always starting with the farthest vantage point (1) and then approaching the corner (Vantage Points 2 and 3 ).

Procedure. The observers were first asked to judge the distance to the corner in question. To provide a standard, two objects in the vicinity were pointed out to them that were $20 \mathrm{~m}$ apart. The observers made a verbal distance judgment in meters. Then, they were asked to convey how obtuse or pointed the angle looked to them, as opposed to what they thought or knew the angle to be.

Right angles are preferred percepts when judging ambiguous 2-D drawings (Kopfermann, 1930). This preference is reflected in verbal angle-setting judgments, which tend to produce biases that prefer $90^{\circ}$ and $45^{\circ}$ angles (Gray \& Regan, 1996). However, analogue methods involving a visual match of angles did not show systematic biases. Hence, nonverbal dependent measures were adopted to reduce this bias. A large dial was used, which consisted of a pol- 


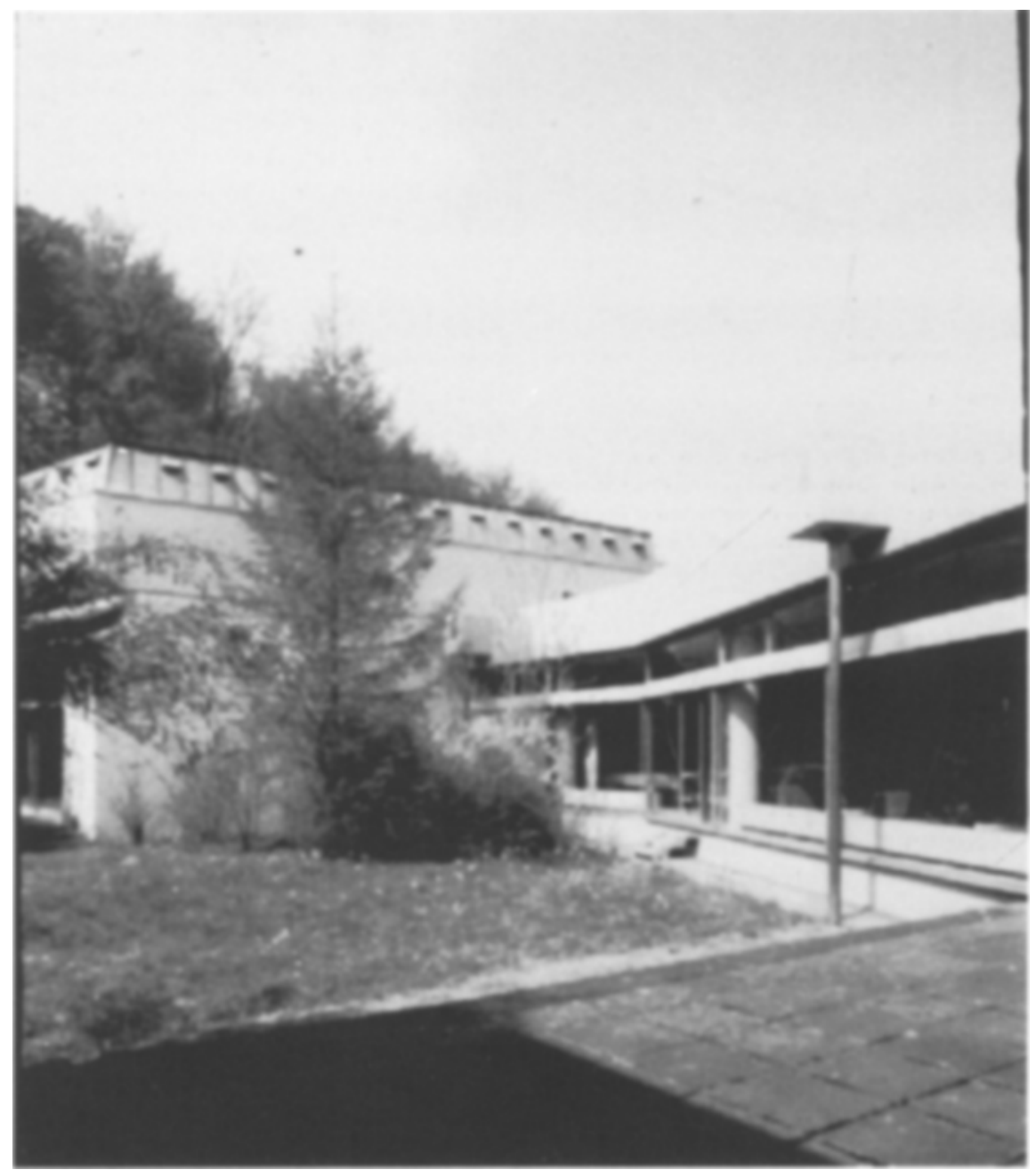

Figure 1. The building corner that observers viewed in the pilot study. To get a better impression of what the real scene looked like, the photograph should be viewed from a distance roughly equal to its width.

ished alumınum disk (diameter, $50 \mathrm{~cm}$ ). On the top side a black line was visible radiating from the center to the edge of the disk. On top of the line was a pointer that had its pivot point at the center of the disk. Like a minute hand of a clock, it could be moved from its starting position in a full circle around the disk. After the observer had moved it so that the angle it subtended with the black line equaled the percelved angle of the corner, the experimenter read off the numerical value of the setting by looking at the bottom side of the dial. The latter was marked with radial lines in $2^{\circ}$ steps and could be read without being visible to the observer. The observers held the dial comfortably in front of them somewhat above their hip, so that the dial surface was parallel to the ground. After each judgment, the dial was handed to the experimenter who read off the setting and moved the pointer back to its resting position.

\section{Results}

The initial impression was confirmed by the pilot observers. On average, the angle subtended by the two corner walls was overestimated by $21^{\circ}$ from the far vantage point. The angle settings are reported in Table 1. Four observers spontaneously reported that they were surprised to see that it was a right angle after all when they moved up close. Only 1 observer failed to recognize the angle as $90^{\circ}$ from the near vantage point. A $t$ test revealed that the angle was significantly overestimated from Vantage Point 1 at a distance of $23.4 \mathrm{~m}[t(6)=3.89, p=.008]$, as well as from Vantage Point 2 at $15 \mathrm{~m}[t(6)=5.00, p=$ $.002]$. Judgments from the near Vantage Point $3(1.5 \mathrm{~m})$ did not differ significantly from $90^{\circ}$.

Distances from the observer to the corner were systematically underestimated by $25.5 \%$ (Vantage Point 1 ), $17.9 \%$ (Vantage Point 2), and $18.0 \%$ (Vantage Point 3). Thus, a misjudgment of distance will certainly not explain the flattening effect between condition 2 and condition 3 . It could conceivably have contributed somewhat to the angle overestimation at the farthest viewpoint.

\section{Discussion}

The initial impression of a strong flattening effect was confirmed by the data. For large viewing distances, the 


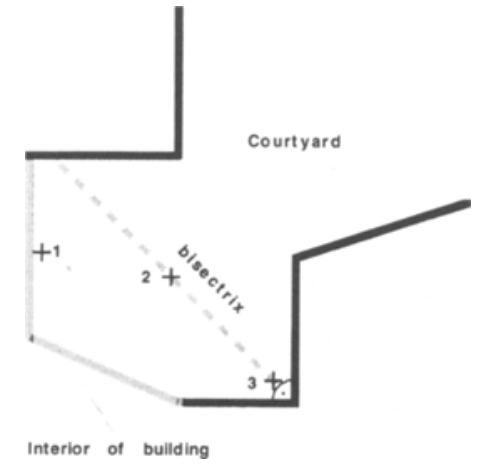

Figure 2. Schematic drawing of the corner used in the pilot study. Solid lines represent walls, shaded lines represent window panes. The first vantage point (1) was $23.4 \mathrm{~m}$ away from the corner. Vantage Points 2 and 3 were at distances of 15 and $1.5 \mathrm{~m}$, respectively.

corner appeared too obtuse, whereas at a $1.5-\mathrm{m}$ viewing distance, all but one observer judged the corner to be a right angle. This result does not seem to be compatible with horopter theory. At a greater than 5-m viewing distance, the hypothetical convex curvature of visual space should lead to an overestimation of distance for objects straight ahead of the observer, whereas objects to either side should appear to be closer. Thus, the vertex of the corner should appear to be farther away, and the angle between the walls should steepen. The opposite was the case. The result is compatible with the more general suggestion of compressed visual space.

However, there are a number of idiosyncrasies associated with this corner. First, the wall to its right bends in the middle (see Figure 2). Thus, if there were a tendency to assume a right angle between the extended parts of the wall, the angle might locally seem to be larger. Second, parts of the walls forming the corner were occluded by a tree and bushes (it looks more severe in the photograph than it did in the real world). We have no explanation as to how occlusion might be responsible for the result, but a better controlled experiment is warranted.

The observer who failed to recognize the corner as a right angle when viewing it close up probably focused on the center part of the walls forming the corner, ignoring the context cues that gave away that it was a right angle. In fact, with some effort, the corner can even be made to invert, similar to a Mach card or a Necker cube, turning the concavity into an apparent convexity.

\section{EXPERIMENT 1}

When looking at a skyscraper from a distance and at some angle, one has the impression that it is flatter than it can possibly be; its visible right angle corner appears to be considerably more obtuse than we know it to be. Thus, there are cases in which we seem to notice a flattening or compression effect for convex corners, at least at very large viewing distances. However, the corner viewed in the pilot study did not, at any point, look strange or even impossible. Experiment 1 was designed to test whether the flattening effect extends to other inconspicuous cases, as to a larger range of corners and vantage points. Also, to rule out the possibility that the experiment tapped into vista space, which the visual system might treat differently than action space (Cutting \& Vishton, 1995), the observer remained within $15 \mathrm{~m}$ of the corner. A building was chosen that provided the opportunity to view various of its corners from sufficiently different vantage points while keeping walking distances between trials at an acceptable level.

If horopter theories are applicable to real-world viewing and if the curvature of the frontoparallel plane is a predominant feature when judging objects that are viewed head-on, concave angles should appear more acute than do convex angles. If, on the other hand, sagittal depth is compressed regardless of the radial position from the observers, all the angles should flatten. Finally, if the pilot results were caused by context effects and if classical space distortions were artifacts of the often used diminished stimuli, angles might, at least on average, be perceived veridically.

\section{Method}

Observers. Eight observers ( 4 male, 4 female) participated in this experiment. Their ages ranged from 21 to 55 years. All had normal or corrected-to-normal vision and were naive with respect to the purpose of the study. Half of them were paid for their participation.

Stimuli and Design. Eight corners were selected from the building complex of the Center for Interdisciplinary Research ( $\mathrm{ZiF})$, which is part of the University of Bielefeld. Three obtuse corners on the outside of the building were chosen. They subtended $108^{\circ}$ (convex), $113^{\circ}$ (concave), and $161.5^{\circ}$ (convex). Three more corners were obtained by viewing the same spots from inside the building, thus creating inside-out corners subtending the same angles. Finally, two outside corners were used that formed right angles (one convex, one concave), resulting in a total of eight different corners. Thus, three corners were inside the building (inside the auditorium, a garage, and a hallway), and five corners were on the outside. Each one was viewed from six different vantage points, with the exception of a few locations that were inaccessible because of spatial limitations. The vantage points were (1) along the bisectrix of the angle subtended by the two adjacent walls forming a corner and (2) displaced by $30^{\circ}$ to the left and (3) to the right. All these positions were

Table 1

Angle Settings for 7 Observers For All Three Vantage Points Used in the Pilot Study

\begin{tabular}{cccc}
\hline Observer & $23.4 \mathrm{~m}$ & $15 \mathrm{~m}$ & $1.5 \mathrm{~m}$ \\
\hline 1 & 100 & 101 & 89 \\
2 & 98 & 101 & 142 \\
3 & 110 & 112 & 95 \\
4 & 108 & 119 & 100 \\
5 & 115 & 125 & 92 \\
6 & 141 & 108 & 87 \\
7 & 106 & 98 & 90 \\
Average & 111 & 109 & $99(92)$ \\
\hline
\end{tabular}

Note-The value in parentheses is the average judgment from the near position excluding the outlier (Observer 2). 


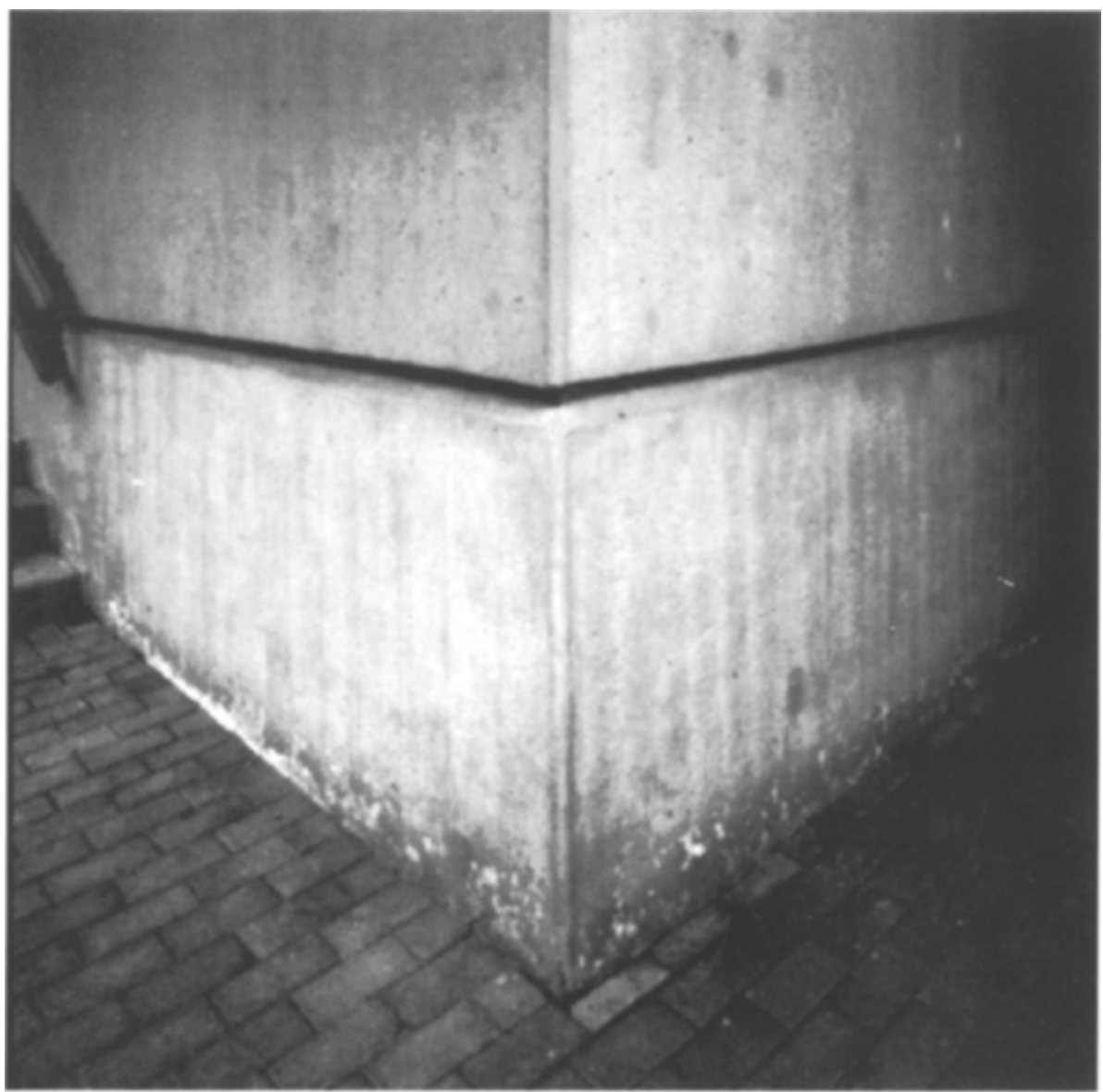

Figure 3. Experiment 1: An exemplar corner as viewed from a distance of $1.5 \mathrm{~m}$. Viewing position is on the bisectrix of this $90^{\circ}$ angle. The figure should be viewed from a distance equal to its width.

fully crossed with two viewing distances. Close viewing positions were always at $1.5 \mathrm{~m}$ distance from the vertex; far positions were $10 \mathrm{~m}$ and more or less, if required by the terrain (one outside corner required a far position of $8 \mathrm{~m}$, another of $5 \mathrm{~m}$, a third of $15 \mathrm{~m}$ ). One of the outside right angle corners is depicted in Figure 3. The photograph was taken from the bisectrix vantage point at $1.5 \mathrm{~m}$ from the vertex.

Procedure All the observers successively looked at all the corners. The sequence of corners varied randomly between observers. For a given corner, one half of the observers always started with the near distance, the others with the far distance. The three vantage points were assumed consecutively but in random order for each cornerdistance combination. From the bisectrix position (see Figure 2), the observers first had to make a distance judgment (from their eye point to the vertex of the angle). Before the first trial, the observers were familiarized with two exemplar distances. They were told that the distance between a particular drainpıpe and a statue in the garden was exactly $20 \mathrm{~m}$. They also were shown a ruler that was $50 \mathrm{~cm}$ long. Subsequently, the observers were asked to convey how obtuse or pointed the angle looked to them, as opposed to what they thought or knew the angle to be. This way, repeated judgments of the same corner from different vantage points seemed justıfied to the observer. For vantage points that were not on the bisectrix, only angle judgments had to be made. The observers communicated their impression of the angle by adjusting the same dial as that used in the pilot study. The whole experiment lasted about $80 \mathrm{~min}$, on average.
The time needed to walk from one corner to the next within the building complex provided ample rest periods between trials.

\section{Results}

As compared with the pilot data, the observers were remarkably accurate. They judged $74 \%$ of the trials within $10^{\circ}$ of the true angle. Only $1 \%$ of the trials deviated by more than $30^{\circ}$. However, as is visible in Figure 4, angles appeared flatter when the observer was farther away, whether the corner was convex or concave. A repeated measures analysis of variance (ANOVA) established that this distance effect was significant $[F(1,7)=$ $14.78, p=.006]$. Neither lateral viewing position nor perceived distance from the corner had an effect on accuracy of judged angle. No significant interactions were found. Distances for the far viewing positions on the bisectrix of the angle were remarkably accurate. An overestimation of near distances was found. On average, the near corners were estimated to be at $2.05 \mathrm{~m}$, instead of the actual $1.5 \mathrm{~m}$, which corresponds to an overestimation of $36.7 \%$. This overestimation was significant $[t(7)=$ $3.50, p=.01]$. Far viewing distances, which ranged from 


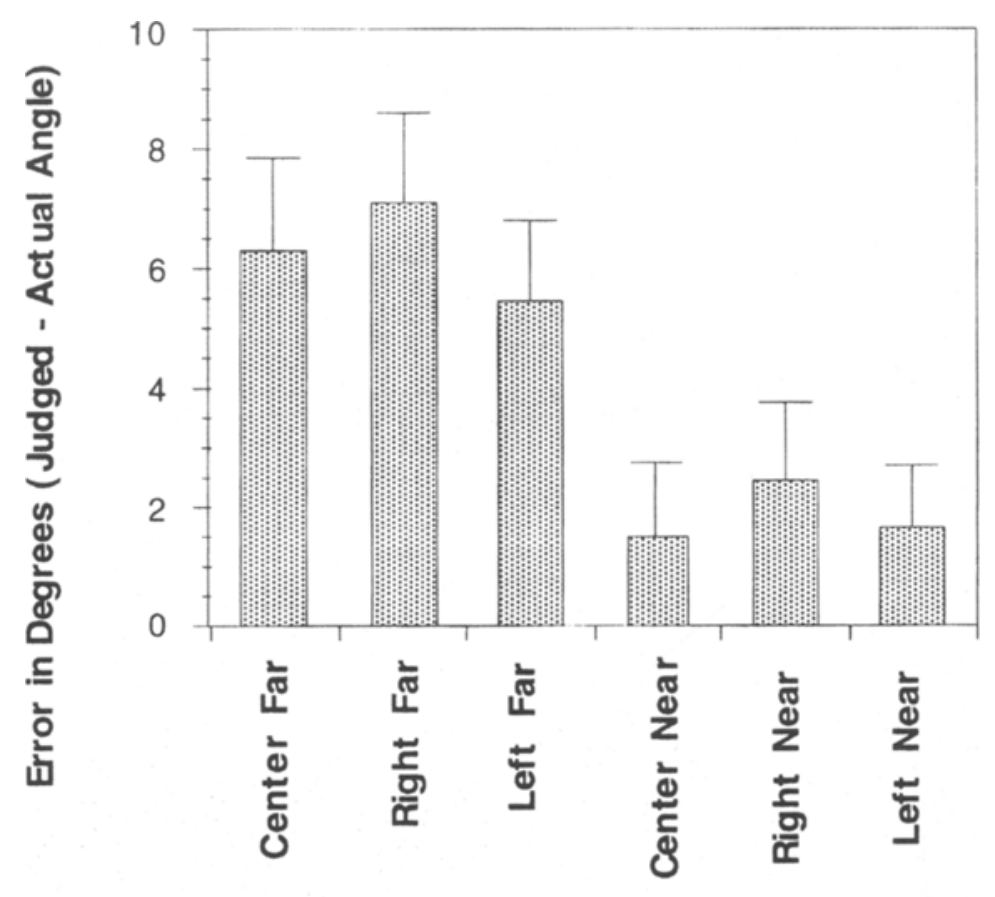

Viewing Posit ion

Figure 4. Average overestimation of an angle in Experiment 1 by viewing distance (near, far) and vantage point (center, right, left). Error bars indicate standard errors of the mean.

5 to $15 \mathrm{~m}$, were overestimated by an average of $7.6 \%$. Those judgments did not differ significantly from the true distance.

\section{Discussion}

All the angles appeared to flatten with larger observer distance. The effect was not as pronounced as that found in the pilot study, but it was very robust. Thus, the effect cannot be attributed to the particular context provided by the adjacent walls or terrain, even though it appears to be enhanced by such context effects. Since only angles of $90^{\circ}$ and more were used, a regression toward the right angle can be ruled out. Likely biases, such as assuming that buildings usually have $90^{\circ}$ corners or an aesthetic preference for right angles, would have worked in an opposite direction from the flattening effect.

The absence of an effect of lateral viewing $\left(30^{\circ}\right)$ suggests that the flattening is not germane to the nongeneric head-on perspective associated with viewing positions along the bisectrix of an angle. Thus, the flattening-withdistance is a general phenomenon that arises beyond viewing distances of $1.5 \mathrm{~m}$. The underestimation of distance to the vertex, relative to the side walls, which was entertained as an explanation of the effect at large viewing distances, is unlikely to be its cause. The observers were able to estimate their distance to the vertex of the corner rather accurately at large distances and, if any- thing, produced an overestimation that should have worked against the flattening effect.

\section{EXPERIMENT 2}

This experiment was conducted to assess whether the flattening effect is preserved or even enhanced in photographic renditions of corners. Photographs provide less resolution, lack stereo cues, and may be taken as 2-D representations. Thus, the corners might look even flatter than in the real world. It is also conceivable that observers are able to compensate for these effects, knowing that pictures always appear less realistic. This argument is supported by findings that observers are often able to extract complex information from pictorial representations and that even toddlers inexperienced with pictures can identify rendered objects (Hochberg \& Brooks, 1962) and extract depth relations from pictorial material (Olson, Yonas, \& Cooper, 1980). Thus, the second experiment addresses the question of whether the spatial relations that were present in our real-world stimuli are preserved in photographs to the extent that the same biases occur.

Experiment 1 was replicated as closely as possible by taking photographs from all the vantage points that were used. Care was taken to always use the same $21-\mathrm{mm}$ lens in the photographic process. When photographs that 
were taken at different focal lengths are then viewed at one distance, magnification or minification effects (Farber \& Rosinski, 1978; Kraft et al., 1986) are likely to occur. A focal length that is too large for a given viewing distance is likely to produce distance overestimation in photographs. If, on the other hand, focal length and distance when viewing the photographs are matched, the focal length is arbitrary. Would-be distortions produced by wide-angle lenses are no better or worse than those produced by "normal" lenses, as long as the respective appropriate viewing distances are assumed. Note that we used a $21-\mathrm{mm}$ lens to achieve a field of view that would approximate natural viewing. During the test, the observers had to maintain the appropriate viewing distance that produced a retinal image maximally similar to what was seen in the natural viewing condition of Experiment 1 . The photographs consisted of black-and-white 35-mm slides. During the experiment, the original slides were displayed on a large rear projection screen, which enabled us to maintain viewing distances of over $1 \mathrm{~m}$ for the observer.

\section{Method}

Observers. The observers ( 9 male, 5 female) were members of the physics department at Utrecht University, who volunteered to participate. They ranged in age from 23 to 60 years. All had normal or corrected-to-normal vision.

Apparatus and Stimuli. Photographs were taken at every vantage point that was used in Experiment 1. The camera (Leica M6) with a $21-\mathrm{mm}$ lens was mounted on a tripod at $1.64 \mathrm{~m}$ above the ground and pointed straight at the vertex of the corner. Black-andwhite portrait format slides were made (on PolaPan $35-\mathrm{mm}$ film, 100 ASA). Durıng the individual experimental sessions, the slides were presented in random order, using a Leitz Pradovit RC projector $(150 \mathrm{~W})$. The image was projected onto the rear surface of a large $(184 \times 119 \mathrm{~cm}$, horizontal $\times$ vertical $)$ translucent projection screen. The observer was seated in front of the screen so that his or her line of sight was directed at the center of the display screen, as well as at the center of the depicted scene. A chinrest was used to keep the line of sight $128 \mathrm{~cm}$ above the ground. The projected scene subtended horizontally a visual angle of $56.4^{\circ}$ and vertically a visual angle of $73.2^{\circ}$. Since the projection was portrait format, whereas the screen was landscape format, the left- and rightmost parts of the screen were not used. Its distance to the eye point of the observer was $112 \mathrm{~cm}$. All the slides were presented in a different random order for each observer.

Procedure. With a remote control, the experimenter advanced the slide projector. The corner in question was pointed out to the observer, and he or she was asked to get an impression for the angle subtended by the two walls forming the corner at the center of the photograph. The projection lamp was the only light source in the room. The same analogue dial as before was used to receive analogue angle judgments. It was held by the observer at arm's length on his or her lap, with a reference line pointed straight ahead. A pointer had to be adjusted. The observers were encouraged to take their time and to double-check theır angle settings. Then, using a flashlight, the experimenter read the settings on the back of the dial and recorded them. On trials corresponding to viewing positions on the angle bisectrix, we asked that distance judgments be made in meters or in fractions thereof. It was pointed out that the diameter of the dial was $0.5 \mathrm{~m}$. On average, the experiment lasted for about $30 \mathrm{~min}$.

\section{Results}

One observer had failed to understand the use of the dial and repeatedly confused concave with convex corners. These data were dropped from the analysis. A repeated measures ANOVA ( 3 viewing positions $\times 2$ distances) was conducted on the constant angle judgment errors made by the remaining 13 observers. Eight trials were dropped from the analysis because several observers had complained that they could not properly see the angles on these photographs. This happened for several far corners, probably owing to the restricted field of view, resolution, and contrast of the images, for one view of the corner already used in the pilot study because of the shrubs partially occluding it, and for one near corner inside the building. One wall of the latter was covered by a wavy curtain, which made it hard to judge the wall's orientation.

As in Experiment 1, angles were significantly overestimated for far, in comparison with near, viewing distances $[F(1,12)=42.43, p<.0001]$. In contrast to the real-world situation, viewing position had a significant effect. Positions on the bisectrix led to more acute angle judgments than did vantage points to the left or to the right $[F(2,24)=12.91, p<.001]$. The interaction of viewing distance and position failed to reach significance $[F(2,24)=3.30, p=.056]$. Unlike before (compare Experiment 1), perceived distance to the vertex of the corner was underestimated by $30.8 \%$ for far vantage points and overestimated by $71.1 \%$ for near vantage points.

To directly test for differences between real-world and photographic viewing, signed judgment errors from Experiments 1 and 2 were entered into one repeated measures ANOVA ( 2 groups $\times 3$ viewing positions $\times 2$ distances). For symmetry reasons, the real-world analogues of the excluded photographs were also not entered into the ANOVA. Figure 5 depicts the average constant judgment errors obtained in both viewing conditions (real world and slide projections onto the big screen). Judged angles were larger for far viewing positions in both photographic and real-world presentations $[F(1,19)=52.60$, $p<.001] .{ }^{3}$ No significant main effect for viewing condition was found $[F(1,19)=2.17, p=.157]$. Viewing position (left, center, right), regardless of distance, made a difference $[F(2,38)=6.85, p=.004]$. This effect was carried by the photographs, as is suggested by the significant interaction between condition and viewing position $[F(2,38)=4.66, p=.019]$ and the absence of a position effect in Experiment 1. Distance interacted neither with position nor with condition.

Post hoc tests showed that real-world judgments did not differ significantly from the true angle for near vantage points, but were significantly larger for far vantage points $[t(7)=5.09, p<.001]$. For photographs taken from far vantage points, angles were likewise overestimated $[t(12)=4.24, p<.001]$. The center near vantage points led to significant underestimations of the angles $[t(12)=$ 


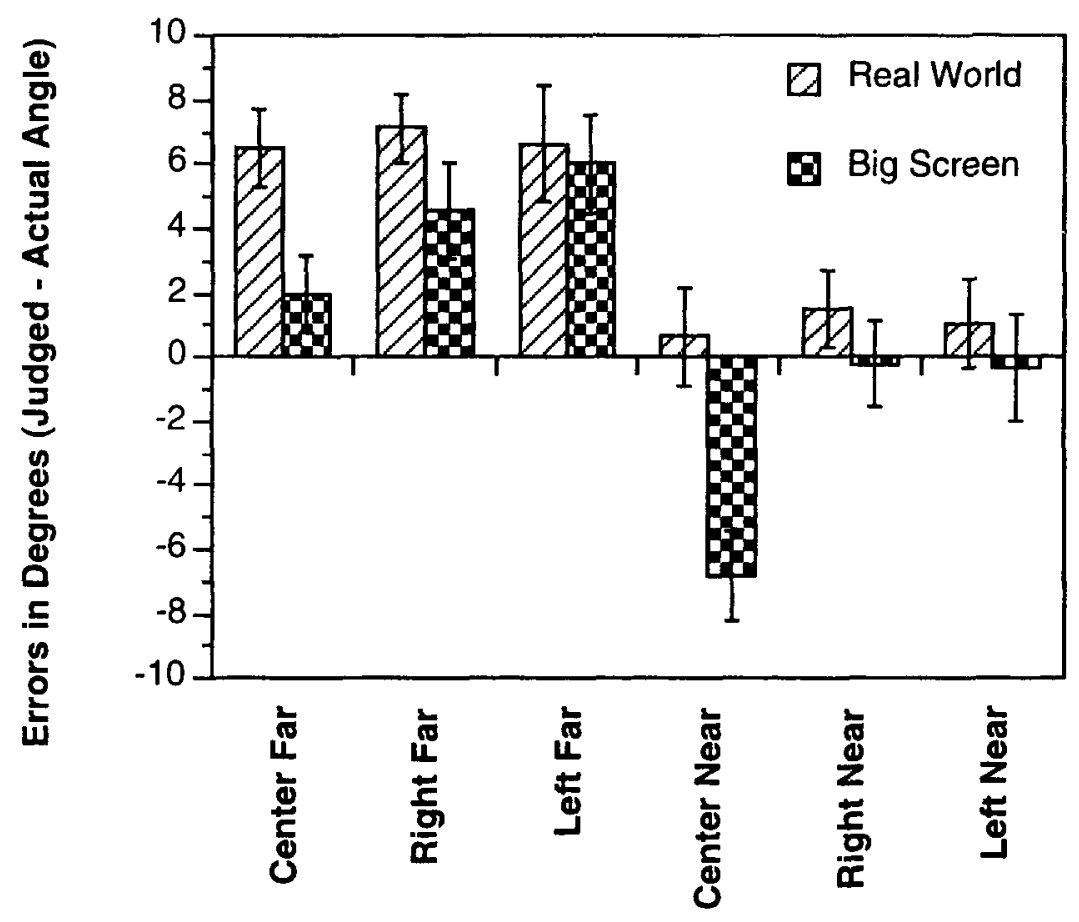

Viewing Position

Figure 5. Average estimation errors for photographic and for real-world stimuli. The latter differ slightly from the results in Experiment 1 because some trials were removed from both conditions, since their photographic rendition was too poor. Positive errors denote an overestimation of angle.

$-3.15, p=.002]$, whereas the estimations from lateral positions did not differ from the accurate estimations.

Performance variability was practically identical to that obtained in the real world: $73 \%$ of all judgments were within $10^{\circ}$ of the true angle, and only $1.6 \%$ of them deviated by more than $30^{\circ}$. A joint repeated measures ANOVA that was conducted on the absolute judgment errors confirmed this impression. The main effect for condition (photograph vs. real world) did not reach significance $[F(1,19)=3.14, p=.092]$.

\section{Discussion}

The flattening effect already found in the real world was replicated for photographic viewing. Looking at the corner from far away $(8-15 \mathrm{~m})$ or seeing photographs taken from the same vantage point led to the impression that the angle was more obtuse. Whereas in the real world, moving close up to the corner (to $1.5 \mathrm{~m}$ distance) cured the misperception and produced, on average, accurate angle judgments, this was not the case in photographs. In particular, corners photographed from the near center position appeared more acute than they actually were. This held for convex and concave corners alike. The effect is not due to outliers; it seems to exist for most corners and for all observers. In Experiment 1, center viewing positions were always assumed first, whereas they were randomized in Experiment 2. However, randomization does not explain why angles of corners are underestimated for near center pictures.

To explain the somewhat strange pictorial effect that corners appear more acute only when photographed from near positions on the bisectrix, two explanations should be considered. First, for oblique viewing angles, the texture gradient of the walls forming the corner is more easily detectable than it is in the head-on view. The relatively large loss of such information at vantage points on the bisectrix might be responsible for the effect. It is conceivable that the reduced pictorial cues for texture and context of close-up corners produced a regression toward right angles. Second, the effect that photographic, but not real, angles appear to become more acute when viewed from near positions on the bisectrix might reflect an influence of the projected retinal image. Side views of a corner lead to differential foreshortening, which might create the impression of a more obtuse angle. A trend supporting the special role of the bisectrix position can also be found for far vantage points and photographic viewing (see Figure 5). The effects may be related to our ability to compensate for no more than the slant of one primary projection surface (Kubovy, 1986; Pirenne, 1970). 
It is not clear why we are immune to such relationships as those found in the projected image only under realworld viewing conditions. Presumably, the perspective cues are overridden by stereo information. Thus, for accurate angle estimation at close range, veridical stereo information might be indispensable.

\section{GENERAL DISCUSSION}

\section{Flattening Effects Are Similar in Real-World and Photographic Viewing}

A flattening effect was found at observer distances of between 5 and $15 \mathrm{~m}$. The angles of vertical building corners were misjudged in a way that is compatible with a subjective compression of visual space. The effect cannot be explained by loss of binocular information or cues to flatness in pictures, since it could be demonstrated for natural viewing as well as for pictures. At far distances, binocular cues lose their efficiency and their role in subjective space (Foley, 1980). Thus, the compression of space found here cannot be attributed to the differential importance of disparity and vergence information at near and far distances. The effect is mainly carried by the scene itself, and not by extraretinal information. Certainly, horopter theories that predict spatial foreshortening on the basis of stereo and vergence cannot explain our results.

We can also rule out the possibility that specific pictorial cues to flatness were effective in the photographic viewing conditions. The typical flatness often found in pictures was not reflected in angle judgments. To the contrary, at close camera distances, the angles were judged to be more acute. A flattening effect owing to optical magnification was reported by Bartley (1951). Two cardboard objects were presented to an unknown number of observers at distances ranging from 10 to $40 \mathrm{in}$. The objects were likened to an opened book and a cube, respectively. Viewing them with binoculars (twofold magnification) led to a flattening effect, as compared with normal viewing. Regrettably, no further details about the experimental setup or the results were reported. Bartley concluded that magnification leads to a flattening effect because it causes less distortion than does a comparable actual approach. That is, given equivalent growth of retinal image size, a slanted object seen through binoculars should produce less perceived slant than its normally viewed counterpart. It is unlikely, however, that Bartley's notion is related to the distance effect we found. Our observers used unaided vision, and the binoculars could have produced a framing effect. It would be interesting to replicate some of the conditions with the use of binoculars, as well as with glasses that frame but do not magnify.

Overall, for large viewing distances, the difference between natural and pictorial viewing is surprisingly small. One of the few findings in the same vein is that withinobject depth relations change little when viewing a torso or an image thereof (Koenderink, van Doorn, \& Kappers, 1995).

\section{Why Are Pictorial Distortions So Small, as Compared With Those Already Found in Natural Viewing?}

The main pictorial effect that was found to be different from natural viewing was the underestimation of angles at near centered camera positions. Experiment 2 ruled out the hypothesis that accommodative cues to flatness are responsible for this effect. Moreover, flattening should have been observed in all the stimuli that were presented on the projection screen, but this was clearly not the case. Similarly, Farber and Rosinski (1978) found that when pictures of objects that were separated in depth (sagittally) were viewed from twice the correct viewing distance, their perceived separation in depth was twice as large, as compared with the canonical viewing position. Likewise, Bengston et al. (1980) found that viewing photographs from incorrectly large distances increased judged pictorial depth. They had observers judge depth in photographs viewed from the wrong distance-that is, too far away from the distance that would be necessary to create an optic array roughly equivalent to what an observer would see if positioned at the lens of the camera. The depth interval between two dolls subtending the same visual angle had to be estimated, as well as the actual size of the dolls. The effects were opposite to our findings with real-world objects: Pictorial distances were overestimated. In contrast to these findings, Cutting (1987) found no effect for physical viewing distance from a computer screen.

Thus, the effect remains rather surprising, especially given the evidence that the viewpoint of pictorial representations has little or no effect on the perception of virtual space when the observer is laterally displaced, but normally becomes important when the wrong distance to the picture is assumed (for a summary, see Goldstein, 1991). However, it would be surprising if there were not some mechanism that compensates for wrong viewing distance that is similar to mechanisms that compensate for oblique viewing angles (Cutting, 1987). Every time we watch a picture taken with a wide-angle lens, we are likely to be too far away from the proper viewpoint-for example, when reading a magazine.

One difference between the pictures taken from the bisectrix and those taken laterally might lie in the cues given by linear perspective, which were rather reduced in center views of corners subtending large angles. One might speculate that, in these cases, the observers regressed toward assuming angles closer to $90^{\circ}$. Or our sample of photographs with centered views might have been biased one way or the other. Be that as it may, the general flattening effect was robust and can be attributed to the camera distance. At the same time, it might be worthwhile to further investigate how robust pictorial angle judgments 
are when made from wrong viewing distances; presumably, they are rather stable.

Compression of pictorial depth in this case also cannot be due to a truncation of the visual field (Hagen et al., 1978; Lumsden, 1983). The flattening effect was just as large with natural viewing, where the field was never truncated. And if anything, the truncation effect should have compensated for flattening effects, since the near camera positions, where visual space was dilated, were the ones with the larger truncations.

Our observers in the real-world viewing condition had their feet planted firmly on the ground but were free to move their shoulders, heads, and eyes. At near viewing distances, they could have obtained some information about the object from motion parallax in addition to binocular disparity. However, this is unlikely, since disparity provides more reliable information than does motion parallax from small head movements (Durgin, Proffitt, Olson, \& Reinke, 1995). At far viewing distances, the natural viewing condition had no advantages over photographic viewing, which indicates that the information in the frozen optic arrays of the pictures that were used was optimally exploited for the task. This finding supports hypotheses about the array-specific information about spatial layout (Gibson, 1971; Halloran, 1989; Warren, 1984).

\section{The Size of the Effect}

Judgment errors, regardless of presentation mode, were around $10 \%$. This puts them in a class with most laboratory studies, and not with outdoors studies. For instance, Wagner's (1985) observers had to judge angles between three randomly positioned stakes at large distances (up to $40 \mathrm{~m}$ ) in a flat grassy terrain. He found a compression of visual space of $50 \%$ and more, which is considerably more than what we obtained. For example, a typical compression parameter of $50 \%$ would lead to an overestimation of a $90^{\circ}$ angle to be $128^{\circ}$. Even the striking angle used in our pilot study was judged to be $111^{\circ}$ on average. It appears that a natural viewing situation does not guarantee that the effects are small, but rather that two factors determine the size of the effect. First, when exocentric, rather than egocentric, distances are involved, judgments are more error prone (Loomis et al., 1996). Second, shallow incident viewing angles produce substantial projective foreshortening that cannot be overcome in pictures or in natural situations (Sedgwick, 1991), even though the slope of regard can provide a cue for absolute distance (Wallach \& O'Leary, 1982). It might be worthwhile to separate the two confounded factors in laboratory experiments in which incidence angle and stereo information are typically correlated.

\section{Comparison With Luneburg's and Gilinsky's Predictions}

Assume a depth function $f(r)$ - that is to say, $r$ is the range and $f(r)$ the perceived depth. Such functions have been proposed by many; two well-known examples are the function from Gilinsky (1951; see also W. M. Smith, 1952), which has also been used by others,

$$
f(r)=\frac{a r}{a+r},
$$

and that from Luneburg $(1947,1950)$ :

$$
f(r)=a e^{-\frac{\delta}{r}} \text {. }
$$

The constant in the Gilinsky function is empirically found to be about $30 \mathrm{~m}$. The constant in the Luneburg function
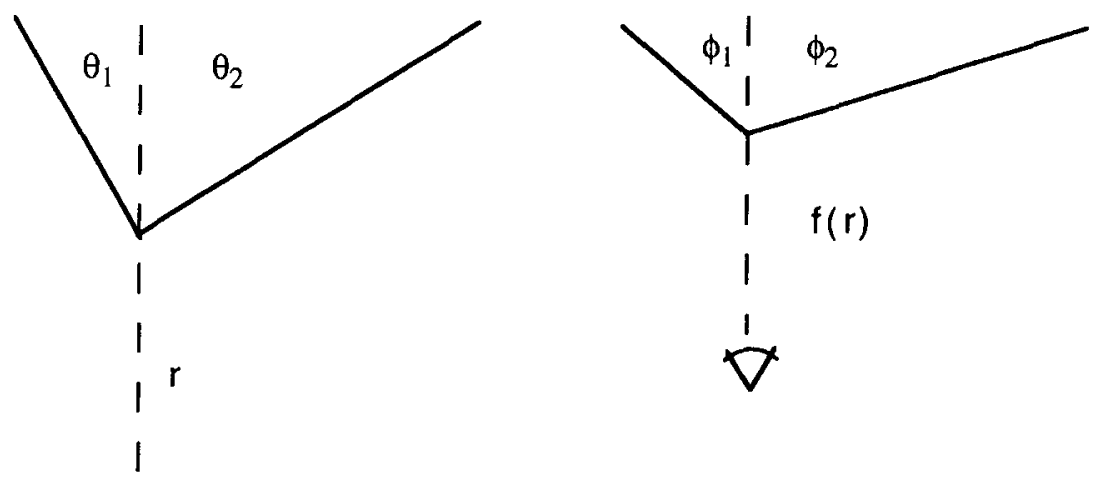

Deformed Perceptual Space

\section{Physical Space}

\footnotetext{
Figure 6. Perceptual space as a function of physical space. Luneburg and Gilinsky describe perceptual space as deformed, in comparison with physical space. For the general case, the line of sight (dashed line) is directed toward the vertex of the corner but does not necessarily halve the angle subtended by the two walls $(\theta)$.
} 
Table 2

Average Judgment Errors Predicted by Luneburg and Gilinsky and Actual Judgment Errors for a Subset of the Conditions Used

\begin{tabular}{cccc}
\hline Viewing Position & Gilinsky & Luneburg & Judgment \\
\hline Right $1.5 \mathrm{~m}$ & 7 & -11 & 7 \\
Right $9.8 \mathrm{~m}$ & 1 & -50 & 3 \\
Right $23.4 \mathrm{~m}$ & 34 & 22 & 21 \\
\hline
\end{tabular}

Note-Positive errors indicate overestimation of angle. Actual judgment errors are averaged for close (top row, from Experiment 1), intermediate (center row, from Experiment 1), and far (bottom row, from pilot study) viewing positions, always to the right of the bisectrix.

is under debate, but a value of $\delta=15 \mathrm{~m}$ yields a result that roughly coincides with the Gilinsky function in the range of the distances of interest in the present experiment.

The nonlinear nature of the depth function leads to a spatial deformation. This can be used to predict the results of angle judgment, if one assumes that the depth function is due to an overall deformation of space. We find that the angle $\theta$, composed of the two angles $\theta_{1}$ and $\theta_{2}$ (see Figure 6), is expected to lead to a perceived angle $\phi=$ $\phi_{1}+\phi_{2}=g\left(\theta_{1}\right)+g\left(\theta_{2}\right)$, where

$$
g(\theta)=\cot ^{-1}\left[r \frac{1}{f} \frac{\partial f}{\partial r} \cot \theta\right]
$$

(the derivative being taken at the distance of the angle). See the Appendix for a derivation. For the Gilinsky (1951) function, this yields

$$
\phi=\cot ^{-1}\left[\frac{a}{a+r} \cot \theta\right]
$$

and, for the Luneburg $(1947,1950)$ function,

$$
\phi=\cot ^{-1}\left[\frac{\delta}{r} \cot \theta\right] \text {. }
$$

Thus, all the angles are predicted to be perceived as being more obtuse than they are in the Gilinsky case, whereas they are predicted to be perceived as being more acute at close range $(r<\delta)$ and more obtuse at far range $(r>\delta)$ for the Luneburg case.

Our data are not fully compatible with either of these predictions. One comparison was made for outdoor viewing and is shown in Table 2 . Since we have only measured one angle at a distance greater than $15 \mathrm{~m}$, which was only viewed from a vantage point to the right of the bisectrix (see the Pilot Experiment section), only judgments made from right ${ }^{4}$ viewing positions were chosen for this comparison. Performance at close viewing distances was better predicted by Gilinsky (1951), whereas Luneburg's $(1947,1950)$ predictions were a better match for the data at far viewing distance. Luneburg's theory can be taken to involve a reversal of error that could point to compression of space at far viewing distances and to dilation of space at near viewing distances.

A comparison that includes pictorial viewing on the large rear projection screen (Experiment 2 ) is depicted in Figure 7. Here, values were calculated on the basis of av- erage judgments and predictions for vantage points on the bisectrix of the angle. For a given viewing distance (abscissa), the hypothetical subjective distance values (ordinate) according to Gilinsky (1951) and Luneburg (1947, 1950) are depicted. Distance judgments obtained in Experiments 1 and 2 are also represented. It can be seen that distance judgments in the real world were closest to veridical perception (Euclidean geometry). The data obtained from big-screen displays are somewhere between the values predicted by Gilinsky and those predicted by Luneburg. We hasten to add that conclusions from this ex post comparison might be premature. More data points at values greater than $15 \mathrm{~m}$ and converging results are needed.

\section{Sagittal Compression for Far Objects, Dilation for Near Objects?}

For near corners, real-world observers were accurate, whereas photographic viewing produced underestimation of angle, especially for centered viewpoints. Horopter experiments, as well as alley stimuli, produce a reversal of effects at very near viewing distances. Typically, perception at distances of around $2 \mathrm{~m}$ is quite accurate, and distortions are opposite for closer and farther distances. Most results could be interpreted as a compression effect for far distances and as a dilation effect for near distances. Accordingly, we would have expected that building corners at the $1.5-\mathrm{m}$ viewing distances used in our near conditions would look more acute than they actually were. This was

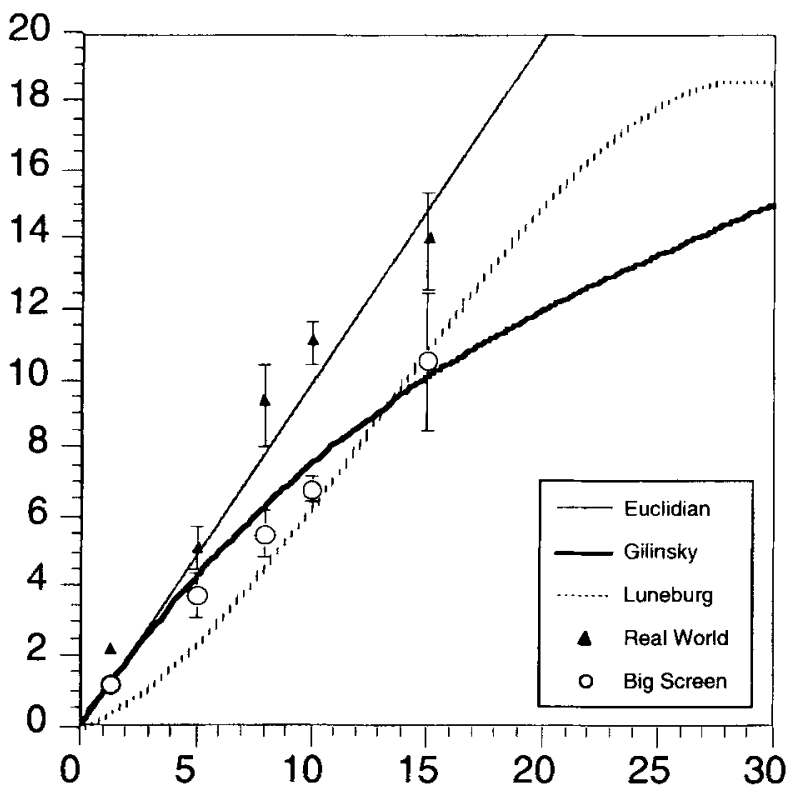

Figure 7. Plot of subjective distance (ordinate) as a function of physical distance (abscissa). Distance judgments from Experiments 1 and 2 are compared with veridically perceived physical space (Euclidean) and with predictions made by Gilinsky (1951) and Luneburg $(1947,1950)$. Error bars indicate standard errors of the mean. They were not drawn when exceeded by the size of the symbol. 
not the case for natural viewing, and more empirical testing is necessary to determine whether such an effect is found for even closer viewing distances. In the photographic condition, on the other hand, results are compatible with a dilation effect. Thus, it is conceivable that there is a general mechanism of sagittal compression for far space and of dilation for near space. This tendency could be overcome by extraretinal information in natural situations where it is relevant for our actions, as it is in our personal space, where we can touch and handle objects.

\section{Do the Results Generalize and Do They Support a Uniformly Distorted Visual Space?}

If subjective space is, in some sense, uniformly distorted, errors in perceived distance should fit together with angular distortion. That is, close angles should steepen, and if the base of the angle is used as reference, the distance from the observer to the vertex should be overestimated in the case of concave corners. In the case of close convex corners, it should be underestimated. At large distances, the whole situation reverses. Against these predictions, we found no effect of convexity for near vantage points in either of the experiments. Far vantage points showed no effect in the big-screen projections, but outdoors there was a trend to judge distances of concave corners to be larger. In Experiment 1, distances of far concave angles were overestimated by $12.4 \%$, and distances of far convex angles were misjudged by $2.5 \%$. This is an almost paradoxical relationship between distance and angle perception, which could indicate that distortion is not uniform or that the angle's base is not always used as reference. Since we collected distance estimates to the apex, but not also to the angle's base, this question cannot be decided at this point.

The finding that, in the real world, distance judgments were fairly accurate suggests that there is no such thing as a subjective space that uniformly affects our perceptual judgments. This is not to say that subjective space is necessarily nonmetric. In particular, we have not investigated any 2-D cues that could have helped or interfered with performance (Lappin \& Love, 1992; Pizlo \& SalachGolyska, 1994). However, our results agree better with contentions that visual space cannot be modeled by any standard geometry (Pizlo, Rosenfeld, \& Weiss, 1997) than they agree with models of metric distortion.

How do our findings relate to models of slant underestimation in reduced viewing conditions, in which the observer is likely to misjudge his or her gaze angle (Perrone \& Wenderoth, 1991)? Such interpretations can be ruled out for three reasons. First, the viewing conditions were not reduced. Second, the angles were symmetric, so that gaze angle was relatively unimportant. And third, oblique views with respect to the bisectrix of the angles did not produce appreciable differences in angle overestimation.

Early papers on the perception of objects at a distance were concerned with the question of whether observers judged the visual world or the visual field (Gibson, 1950; Gilinsky, 1955). Depending on the instructions, they can judge one or the other, which leads to enormous differ- ences. In our experiment, the instructions were meant to suggest a visual world perspective while not allowing cognitive aspects to enter. Several subjects asked whether they should say something other than $90^{\circ}$, even though they knew building corners were right angles. In this case, we told them the truth-that is, we informed them that the $\mathrm{ZiF}$ building was funny and that, in fact, less than half of all the angles were right angles, so they should by all means go with what they saw.

The stimuli that we used were rather untypical for experiments of spatial distortion. With their use, we were able to reinterpret some of the classical findings, and it appears to be worthwhile to extend the class of stimuli considered in the study of visual space. Also, since the evidence is growing that space may not be represented in a uniformly distorted manner, different objects are likely to affect subjective space differently and need to be studied.

In conclusion, visual space seems to be represented as accurately as is necessary in personal and close action space, in Cutting and Vishton's (1995) terminology. Where accuracy is not crucial, errors enter. These errors can be described as a compression of depth and might be a function of spatial representation. We need only perceive far action space and vista space well enough to build representations that allow us to recognize and locate objects that could or are about to enter our personal space. These representations are equally well accessed by real-world viewing and photographic viewing. In both cases, perspective problems enter and are particularly serious when stimuli are presented on a horizontal plane (Indow, 1991). In personal space, real-world viewing conditions do not suffer from the same distortions as do pictorial representations.

\section{REFERENCES}

Ames, A., OGle, K. N., \& Glidden, G. H. (1932). Corresponding ret1nal points, the horopter and the size and shape of ocular images. Journal of the Optical Society of America, 22, 631-638.

BARTLEY, S. H. (1951). A study of the flattening effect produced by optical magnification. American Journal of Optometry, 28, 290-299.

Battro, A. M., Di Pierro Netto, S., \& Roz.estraten, R. J. A. (1976). Riemannian geometries of variable curvature in visual space: Visual alleys, horopters, and triangles in big open fields. Perception, 5, 9-23

Bengston, J. K., Stergios, J. C., Ward, J. L., \& Jester, R E. (1980) Optic array determinants of apparent distance and size in pictures Journal of Experimental Psychology: Human Perception \& Performance, 6, 751-759

Blank, A. A. (1961). Curvature of binocular visual space An experiment. Journal of the Optical Society of America, 51, 335-339

BlumENFELD, W. (1913). Untersuchungen über die scheinbare Größe im Sehraume [Investigation of the apparent size in visual space] Zeitschrift für Psychologie, 65, 241-404.

Bralinstein, M (1968). Motion and texture as sources of slant information. Journal of Experimental Psychology, 78, 247-253

CutTing, J. E. (1987). Rigidity in cinema seen from the front row, side aisle. Journal of Experimental Psychology: Human Perception \& Performance, 13, 323-334.

CutTing, J. E., \& Vishton, P. M. (1995). Perceiving layout and knowing distances: The integration, relative potency, and contextual use of different information about depth. In W. Epstein \& S. Rogers (Eds.), Perception of space and motion (pp. 69-117). New York: Academic Press.

Drösler, J., \& Konstanty, R. (1987). Experımentelle Untersuchung der Geometrie des monokularen Sehraumes [Experimental invest1gation of the geometry of monocular visual space]. Zeitschrift für Experimentelle und Angewandte Psychologie, 34, 351-369. 
Durgin, F. H., Proffitt, D. R., Olson, T. J., \& Reinke, K. S. (1995). Comparing depth from motion with depth from binocular disparity. Journal of Experimental Psychology: Human Perception \& Performance, 21, 679-699.

Eby, D. W., \& BraUnsteIN, M. L. (1995). The perceptual flattening of three-dimensional scenes enclosed by a frame. Perception, 24, 981-993.

Ehrenstein, W. H. (1977), Geometry in visual space: Some methoddependent (art1)facts. Perception, 6, 657-660

Ellis, S. R, \& Hacisalihzade, S. S. (1990, October). Symbolic enhancement of perspective displays. Proceedings of the 34th Meeting of the Human Factors Society (pp. 1465-1469). Santa Monica, CA; Human Factors Society.

El.tis, S. R., Smith, S., Grunwald, A., \& McGreevy, M. W. (1991). Direction judgment error in computer generated displays and actual scenes. In S. R. Ellis, M. K. Karser, \& A. C. Grunwald (Eds.), Pictorial communication in virtual and real environments (pp. 496-503). London: Taylor \& Francis.

FARBER, J., \& Rosinski, R. R. (1978). Geometric transformations of pictured space. Perception, 7, 269-282.

Flock, H. R. (1964). A possible optical basis for monocular slant perception. Psychological Review, 71, 380-391.

Foley, M. (1972). The size-distance relation in intrinsic geometry of visual space. Vision Research, 13, 323-332.

FolEY, M. (1977). Effect of distance information and range on two indices of visually perceived distance. Perception, 6, 449-460.

Foley, M. (1980). Binocular distance perception. Psychological Review, 87, 411-434

Frr, G. (1950). Visual perception of space. American Journal of Optometry, 27, 531-553.

GiBson. J. J. (1950). The perception of the visual world. Boston: Houghton Mifflin.

Gibson, J. J. (1954). A theory of pictorial perception. Audio-Visual Communications Review, 1, 3-23.

GiBSON, J. J. (1971). The information avallable in pictures. Leonardo, 4, 27-35.

Gibson, J. J.. \& Cornsweet, J. (1952). The perceived slant of visual surfaces optical and geographical Journal of Experimental Psychology, 44, $11-15$.

GILINSKY. A. S. (1951). Perceived size and distance in visual space. Psychological Review, 58, 460-482.

GILINSKY, A. S. (1955). The effect of attitude upon the perception of size. American Jourral of Psychology, 68, 173-192.

Gil. am, B., \& Ryan, C. (1992). Perspective, orientation disparity, and anisotropy in stereoscopic slant perception. Perception, 21, 427-439

GoGEL. W. C. (1964). Visual perception of spatial extent. Journal of the Optical Society of America, 54, 411-416.

GOGEL, W. C. (1965). Equidistance tendency and its consequences. Psychological Bulletin, 64, 153-163

Gogel. W. C. (1993). The analysis of perceived space. In S. C. Masin (Ed.), Foundations of perceptual theory (pp. 113-181). Amsterdam: Elsevier.

Goldstein, E. B. (1991). Perceived orientation, spatial layout and the geometry of pictures. In S. R. Ellis, M. K. Kaiser, \& A. C. Grunwald (Eds.), Pictorial communication in virtual and real environments (pp. 480-485). London: Taylor \& Francis.

Gray, R., \& REgan, D. (1996). Accuracy of reproducing angles. Is a right angle special? Perception, 25, 531-542.

HaGEN, M. A. (ED.) (1980). The perception of pictures. Vol I Albertis window. The projective model of pictorial information. New York: Academic Press.

Hagen, M. A., Jones, R. K., \& ReEd, E. S. (1978). On a neglected variable in theories of pictorial perception: Truncation of the visual field. Perception \& Psvchophysics, 23, 326-330.

Halloran, T. O. (1989). Picture perception is array-specific: Viewing angle versus apparent orientation. Perception \& Psychophysics, 45, $467-482$.

Hauck, G. (1875). Die subjektive Perspektive und die horizontalen Curvaturen des Dorischen Stils [Subjective perspective and the horizontal curvatures of the Doric style]. Stuttgart.

HEEL AN, P. A. (1983). Space-perception and the philosophy of science. Berkeley: University of California Press.
Helmholtz, H. von (1867). Handbuch der physiologischen Optik [Handbook of physiological optics]. Leipzig: Voss.

HoChBerg, J., \& Brooks, V. (1962). Pictorial recognition as an unlearned ability: A study of one child's performance. American Journal of Psychology, 75, 624-628.

Howard, I. P., \& Rogers, B. J. (1995). Binocular vision and stereopsis. Oxford: Oxford University Press.

INDOw, T. (1991). A critical review of Luneburg's model with regard to global structure of visual space. Psychological Review, 98, 430-453

InDOw, T. (1997). Hyperbolic representation of global structure of visual space. Journal of Mathematical Psychology, 41, 89-98.

InDow, T., Inoue, E., \& Matsushima, K. (1962a). An experimental study of the Luneburg theory of binocular space perception: 1 . The 3- and 4-point experiments. Japanese Psychological Research, 4, 6-16.

Indow, T., Inoue, E., \& Matsushima, K. (1962b). An experimental study of the Luneburg theory of binocular space perception: 2 . The alley experiments. Japanese Psychological Research, 4, 17-24.

InDOW, T., \& WaTANaBE, T. (1988). Alleys on an extensive apparent frontoparallel plane: A second experiment. Perception, 17, 647-666.

JamES, W. (1950). The principles of psychology (Vol. 2). New York: Dover. (Original work published 1890 )

Koenderink, J. J., van Doorn, A. J., \& Kappers, A. M. L. (1995) Depth relief. Perception, 24, 115-126.

KoEnderink, J. J., VAN DOORN, A. J., \& LAPPIN, J. S. (1996), Exocentric pointing in the natural environment. Manuscript submitted for publication.

KoPfERmanN, H. (1930). Psychologische Untersuchungen über die Wirkung zweidimensionaler Darstellungen körperlicher Gebilde [Psychological investigations of the effect of two-dimensional renditions of bodily objects]. Psychologische Forschung, 13, 293-364.

Kraft, R. N., Patterson, J. F., \& Mitchell, N. B. (1986). Distance perception in photographic displays of natural settings. Perceptual \& Motor Skills, 62, 179-186.

Kubovy, M. (1986). The psychology of perspective and Renaissance art. Cambridge: Cambridge University Press.

LAPPIN, J. S., \& Love, S. R. (1992). Planar motion permits perception of metric structure in stereopsis. Perception \& Psychophysics, 51, 86102.

Loomis, J. M., Da Silva, J. A., Philbeck, J. W., \& Fukusima, S. S. (1996). Visual perception of location and distance. Current Directions in Psychological Science, 5, 72-77.

LuKas, J. (1983). Visuelle Frontalparallelen: Ein Entscheidungsexperiment zu den Theorien von Blank, Foley und Luneburg [Visual frontoparallels: A crucial experiment to test the theories of Blank, Foley, and Luneburg]. Zeitschrift für Experimentelle und Angewandte Psychologie, 30, 610-627.

LUKAS, J. (1996). Psychophysik der Raumwahrnehmung [The psychophysics of space perception]. Weinheim: Beltz Psychologie VerlagsUnion.

LUMSDEN, E. A. (1980). Problems of magnification and minification: An explanation of the distortions of distance, slant, shape, and velocity. In M. A. Hagen (Ed.), The perception of pictures. Vol I Alberti's window: The projective model of pictorial information (pp. 91-135). New York: Academic Press.

LUMSDEN, E. A. (1983). Perception of radial distance as a function of magnification and truncation of depicted spatial layout. Perception $\&$ Psychophysics, 33, 177-182.

LUNEBURG, R. K. (1947). Mathematical analysis of binocular vision. Princeton, NJ: Princeton University Press.

LUNEBURG, R. K. (1950). The metric of binocular visual space. Journal of the Optical Society of America, 40, 627-642.

OgLe, K. N. (1950). Researches in binocular vision. Philadelphia: Saunders.

Olson, R., Yonas, A., \& CoOper, R. (1980). Development of pictorial perception. In M. A. Hagen (Ed.), The perception of pictures. Vol 2 Dürer's devices. Beyond the projective model of pictures (pp. 155-192). New York: Academic Press.

Perrone, J. A., \& Wenderoth, P. (1991). Visual slant underestimation. In S. R. Ellis, M. K. Kaiser, \& A. C. Grunwald (Eds.), Pictorial communication in virtual and real environments (pp. 496-503). London: Taylor \& Francis. 
Philbeck, J. W., Loomis, J M., \& Beal.t, A. C. (1995, May). Measurement of visually perceived egocentric distance under reducedand full-cue conditions. Paper presented at the meeting of the Association for Research in Vision and Ophthalmology, Sarasota, FL.

Pirenne, M. A. (1970). Optics, painting \& photography. Cambridge: Cambridge University Press.

Pizlo, Z., Rosenfel.D, A., \& Weiss, I. (1997). The geometry of visua! space: About the incompatibility between science and mathematics. Computer Vision \& Image Understanding, 65, 425-433.

Pizlo, Z., \& SalaCh-GolySKa, M. (1994). Is vision metric? Comment on Lappın and Love. Perception \& Psychophysics, 55, 230-234.

Proffitt, D. R., Bhali A, M., Gossweiler, R., \& Midgett, J. (1995). Percerving geographical slant. Psychonomic Bulletin \& Review, 2, 409-428.

Ross, H. E. (1974). Behavior and perception in strange environments. London: George Allen \& Unwin.

SEDGWICK, H. A. (1991). The effects of viewpoint on the virtual space of pictures. In S. R. Ellis, M. K. Kaiser, \& A. C. Grunwald (Eds.), Pictorial communication in virtual and real environments (pp. 460479). London: Taylor \& Francis.

SMITH, O. W (1958). Judgments of size and distance in photographs. American Journal of Psychology, 71, 529-538.

SмIтн, R. (1738). A compleat system of opticks with J. Jurin's Essay upon distinct and indistinct vision. Cambridge.

SMiTh, W. M. (1952). Gilınsky's theory of visual size and distance. Psychological Review, 59, 239-243.

SuPPES, P. (1977). Is visual space Euclidean? Synthèse, 35, 397-421.

WAGNER, M. (1985). The metric of visual space. Perception \& Psychophysics, 38, 483-495.

WALLACH, H., \& O'LEARY, A. (1982). Slope of regard as a distance cue. Perception \& Psychophysics, 31, 145-148.

WARREN, W. H. (1984). Perceiving affordances: Visual guidance of stair climbing. Journal of Experimental Psychology: Human Perception \& Performance, 10, 683-703.

Watanabe, T. (1996, September). Elliptic walking space and hyperbolic visual space. Poster presented at European Conference on Visual Perception, Strasbourg, France. (Reprinted in Perception, 25[Suppl.] 132.)

YounG, M. J., LANDY, M. S., \& Maloney, L. T. (1993). A perturbation analysis of depth perception from combinations of texture and motion cues. Vision Research, 33, 2685-2696.

ZAJACZKOWSKA, A. (1956). Experimental test of Luneburg's theory: Horopter and alley experiments. Journal of the Optical Society of America, 46, 514-527.

\section{NOTES}

1. Howard and Rogers (1995) claim that the Hering-Hillebrand deviation found in a typical horopter apparatus can be explained by the absence of vertical disparities.

2. A notable exception can be found in the work of Heelan (1983), who attempted to apply the philosophy of science to the notion of subjective space by adding cognitive aspects to hyperbolic distortion functions.

3. The $p$ values are Greenhouse-Geisser corrected.

4. As is visible in Figure 4, left and center viewing positions were very similar to the right vantage points.

\section{APPENDIX}

Consider the infinitesimal rectangle $\mathrm{ABCD}$, where $\mathrm{AD}=$ $r \delta \phi$ and $\mathrm{AB}=\delta r$. Here, $r$ is the range, $\delta r$ an inf initesimal range increment, and $\delta \phi$ an infinitesimal angle. The diagonal $\mathrm{AB}$ has a slant (the angle $\mathrm{BAC}$ ) $\theta$, so that

$$
\cot \theta=\frac{\mathrm{AB}}{\mathrm{BC}}=\frac{\delta r}{r \delta \phi} .
$$

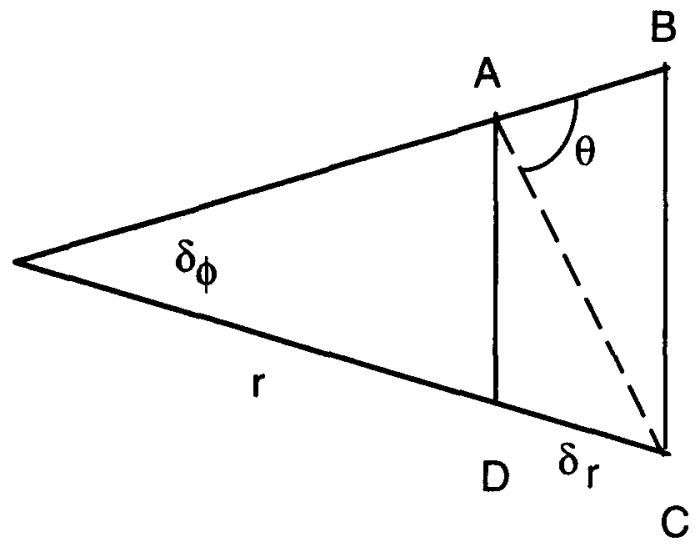

Consider a depth function $f(r)$. Now the geometry is changed; the image of the rectangle is $A^{\prime} B^{\prime} C^{\prime} D^{\prime}$.

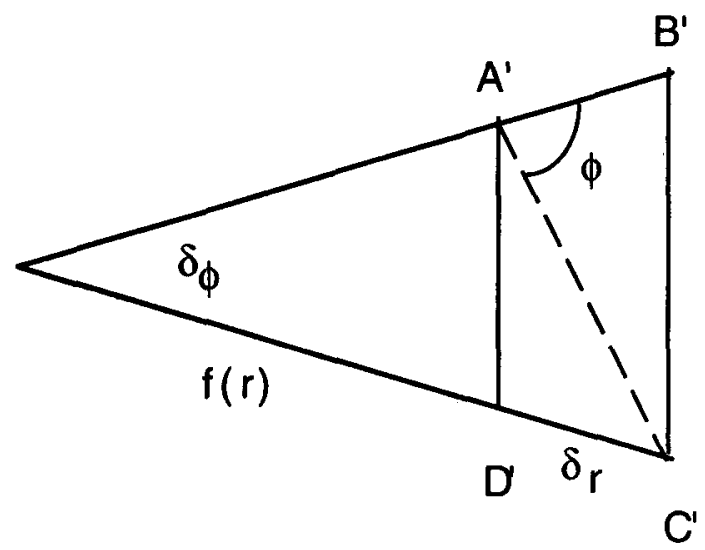

The infinitesimal angle $\delta \phi$ is not changed, but the range $r$ is replaced with the depth $f(r)$. Thus, the side $A^{\prime} D^{\prime}$ becomes $\delta \phi f(r)$, and the side $\mathrm{A}^{\prime} \mathrm{B}^{\prime}$ becomes

$$
\frac{\partial f}{\partial r} \delta r
$$

where the derivative of the depth function

$$
\frac{\partial f}{\partial r}
$$

is to be evaluated at $r$. The angle $\phi$ is given through

$$
\cot \phi=\frac{\mathrm{A}^{\prime} \mathrm{B}^{\prime}}{\mathrm{B}^{\prime} \mathrm{C}^{\prime}}=\frac{\frac{\partial f}{\partial r} \delta r}{f(r) \delta \phi}={ }_{f}^{r} \frac{\partial f}{\partial r} \cot \theta .
$$

Thus, we find the "subjective" slant $\phi$ in terms of the "objective" slant $\theta$ as:

$$
\phi=\cot ^{-1}\left[r \frac{1}{f} \frac{\partial f}{\partial r} \cot \theta\right],
$$

which is the expression used in the text.

(Manuscript received July 7, 1997; revision accepted for publication July 27,1998 .) 